\title{
W Charge Asymmetry Measurement in CDF Run 2
}

\author{
C. Issever for the CDF collaboration \\ University of California, Physics Department, Santa Barbara, CA 93106
}

\begin{abstract}
We present the status of the forward-backward charge asymmetry measurement for $W$ boson production using early Run 2 data collected with the Collider Detector at Fermilab (CDF). Tracking for forward electrons is a critical component of this measurement, and we describe a new technique which combines the position and energy measurements from the calorimeter with position measurements in the silicon detector to provide tracking and charge determination for electron candidates. The performance of this algorithm is described and the sensitivity for the $W$ charge asymmetry measurement with Run 2 data is quantified.
\end{abstract}

\section{INTRODUCTION}

Measurement of the forward-backward charge asymmetry in $p \bar{p} \rightarrow W^{ \pm}$provides a constraint on the parton fluxes within the proton. Since $u$ quarks carry, on average, a higher fraction of the proton momentum than $d$ quarks, the $W^{+}$in $u \bar{d} \rightarrow W^{+}$tends to be boosted in the proton direction. Similarly, a $W^{-}$tends to be boosted in the anti-proton direction. This results in an expected non-zero forward-backward charge asymmetry defined to be

$$
A\left(y_{W}\right)=\frac{d \sigma\left(W^{+}\right) / d y-d \sigma\left(W^{-}\right) / d y}{d \sigma\left(W^{+}\right) / d y+d \sigma\left(W^{-}\right) / d y},
$$

where $y$ is the rapidity of the $W$ bosons and $d \sigma\left(W^{+,-}\right) / d y$ is the differential cross section for $W^{+}$or $W^{-}$boson production ${ }^{1}$.

Leptonic decays of the $W$ boson provide a cleanly identified sample for studying this asymmetry. Here we consider the electron mode, $W^{ \pm} \rightarrow e^{ \pm} v$. Because the neutrino escapes detection, the rapidity of the $W$ bosons is not directly measurable and the $e^{ \pm}$ direction is used instead. Furthermore, the pseudo-rapidity $\eta$ is used to provide a simple but good approximation of the rapidity ${ }^{2}$. The forward-backward lepton asymmetry,

$$
A\left(\eta_{l}\right)=\frac{d \sigma\left(e^{+}\right) / d \eta-d \sigma\left(e^{-}\right) / d \eta}{d \sigma\left(e^{+}\right) / d \eta+d \sigma\left(e^{-}\right) / d \eta}
$$

provides an experimental observable which convolves the $W$ production asymmetry with the V-A decay distribution. $A\left(\eta_{l}\right)$ (and also $A\left(y_{W}\right)$ ) is sensitive to the ratio of the parton

\footnotetext{
1 The rapidity is defined by $y=\frac{1}{2} \ln \left(\frac{E+p_{z}}{E-p_{z}}\right)$ with the four-momentum of the particle $p=\left(E, p_{x}, p_{y}, p_{z}\right)$.

2 The coordinate system is such that the polar angle $\theta$ is measured from the proton direction, the azimuthal angle $\phi$ is measured from the Tevatron plane and the pseudo-rapidity is defined as $\eta=-\ln (\tan (\theta / 2))$.
} 


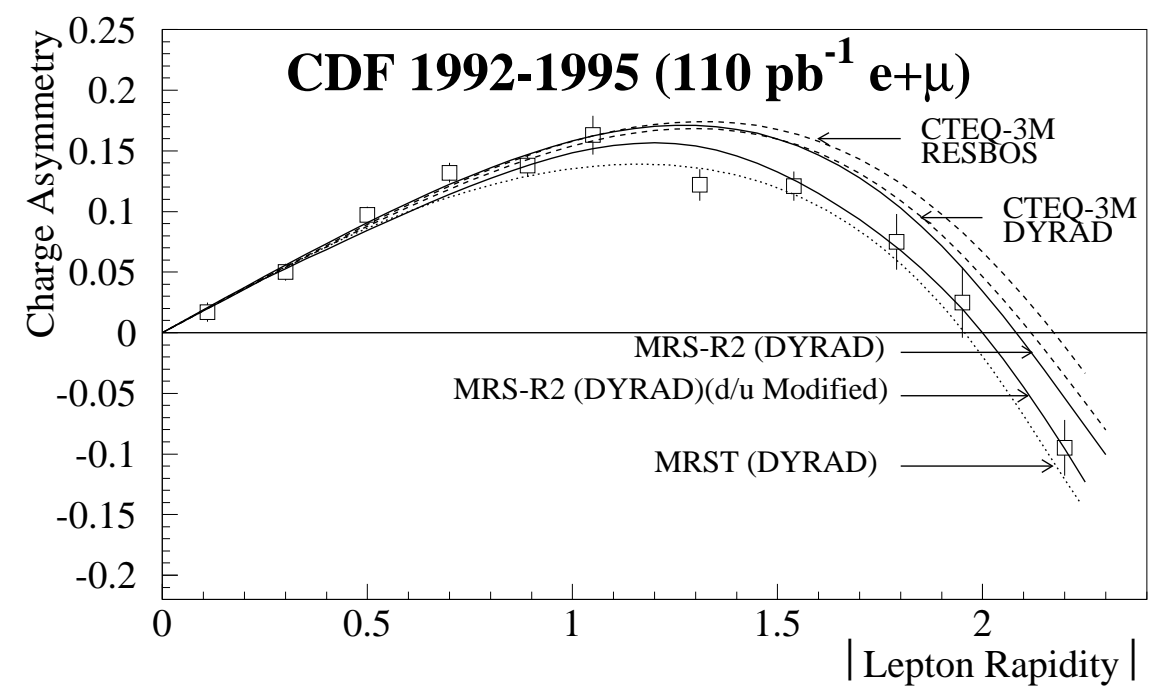

FIGURE 1. CDF's lepton charge asymmetry measurement from Run 1 is compared to the predictions of various parton distribution functions as a function of lepton rapidity. The discrimination is strongest at large values of |LeptonRapidity|[1].

density functions for $u$ and $d$ quarks, $u(x) / d(x)$. This sensitivity is most pronounced at high values of $|\eta|$ (forward region) as shown in Fig. 1

Thus the primary experimental challenge in this measurement is tracking in the forward region to obtain $e^{ \pm}$charge identification. We describe below a newly developed algorithm which uses CDF's extended silicon tracking coverage to reconstruct electron trajectories in the forward direction. This approach is applicable to many electron based measurements in addition to this first use for the $W$ charge asymmetry measurement. We quantify the sensitivity for the asymmetry measurement with the early data from Run 2 and extrapolate the sensitivity to the larger data samples which will be collected soon.

\section{THE CDF RUN 2 DETECTOR}

The Collider Detector at Fermilab underwent a major upgrade program for Run 2 which is described in detail elsewhere[2]. The features which are particularly relevant to this analysis are a new, scintillating tile based, end-plug calorimeter, a multi-wire drift chamber (COT), and a substantially extended silicon tracking system.

Electrons are identified by energy deposition in the calorimeters which measure energy in separate electromagnetic (EM) and hadronic (HAD) sections. Position sensitive detectors (PES) located in the EM section at the expected maximum shower depth measure the position of electron candidates with a precision of about $1 \mathrm{~mm}$. Calorimeter based selection requirements are applied to electron candidates to reject jet backgrounds, but to significantly reduce the backgrounds track matching is needed.

The momentum and charge of final state particles are precisely measured by track 


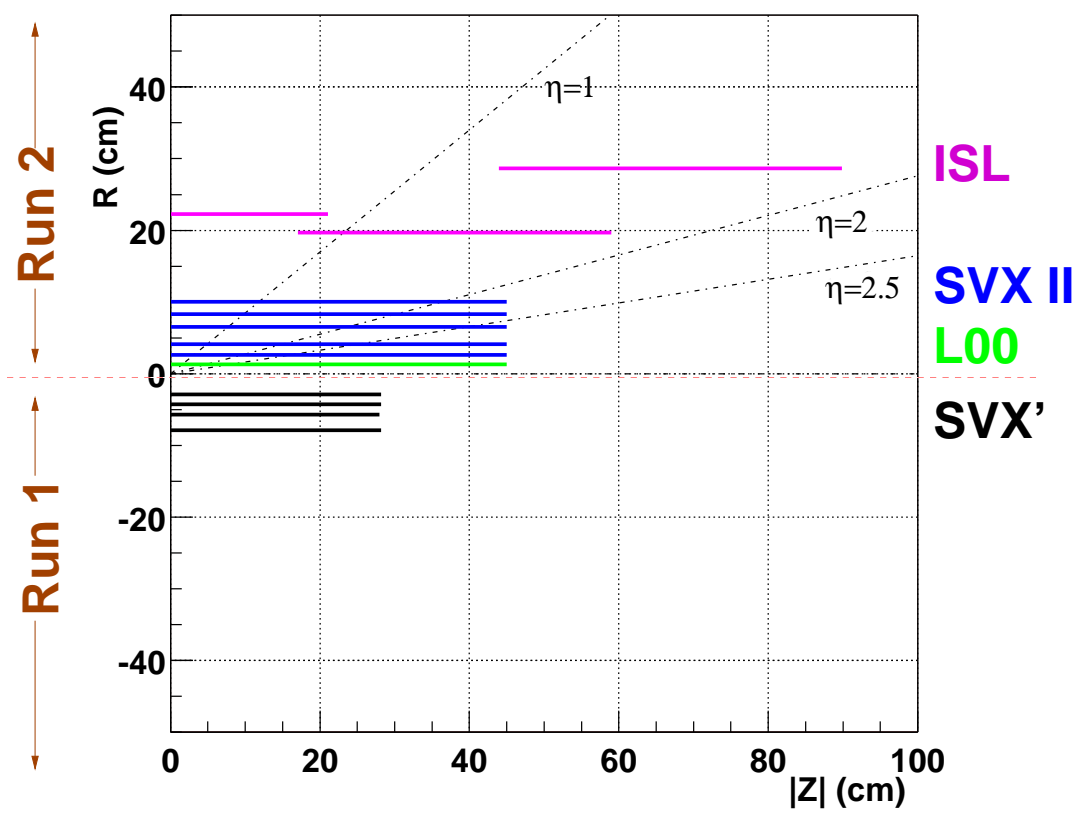

FIGURE 2. Comparison of the Run 2 and Run 1 silicon detector in a schematic rz-view. Lower part: The Run 1 Silicon Vertex Detector (SVX') with 96 single-sided ladders. Upper part:Run 2 silicon detector consisting out of three subdetectors: Layer 00 (L00) a single-sided layer of 48 ladders mounted directly on the beampipe, which enhances the impact parameter resolution. The Silicon Vertex Detector (SVX II) with 360 double-sided ladders and the Intermediate Silicon Layers (ISL) with 296 double-sided ladders are providing precise hits over a large lever arm out to $|\eta| \simeq 2$ and three dimensional tracking.

reconstruction in the COT, but its acceptance is limited to $|\eta|<1$. Track reconstruction in the higher $|\eta|$ region is provided by the silicon system. It consists of 8 measurement layers spanning a radial region from $1.3 \mathrm{~cm}$ to $28 \mathrm{~cm}$. As shown in Fig. 2, the coverage is substantially increased relative to Run 1 . The large radial lever arm and length provide precision tracking out to $|\eta| \sim 2$. This nearly doubles the tracking coverage from the COT region and provides charge identification in the region which is important for this measurement.

\section{CALORIMETER SEEDED SILICON TRACKING}

To fully exploit the forward coverage of the silicon tracking system, we have developed a calorimeter-seeded silicon tracking algorithm which reconstructs the trajectories of electrons without the COT. In the central region, tracks reconstructed with the COT are improved by the addition of silicon hit information. The COT tracks are projected into the silicon where hits in a narrow road around the projection are considered for addition to the fit. Similarly, the new algorithm uses information from the calorimeter to seed the silicon track reconstruction. It is also possible to reconstruct tracks using only the silicon information. That capability is important for tagging heavy flavor decays in the forward direction, but seeding the fit with calorimeter or COT information is more robust. 


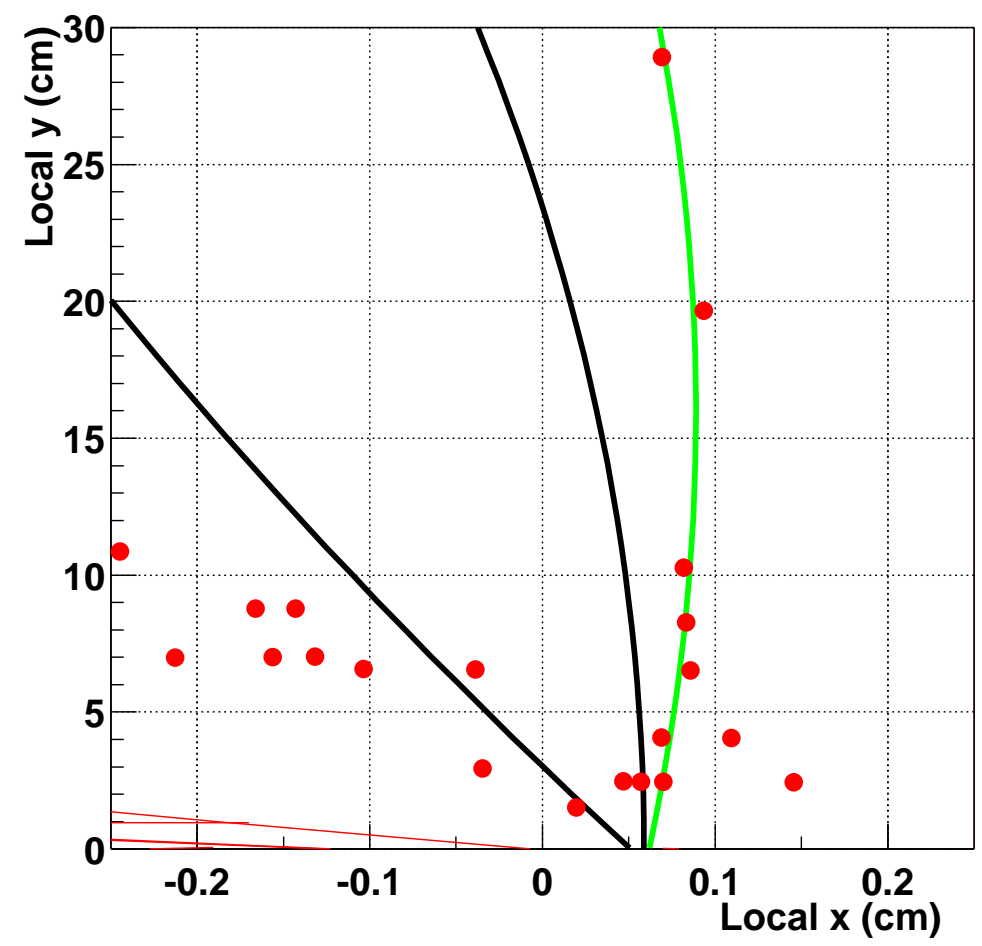

FIGURE 3. An example of a lepton in the forward region from data where one calorimeter seeded silicon track is reconstructed. Shown is the xy-view of the silicon detector with silicon hits (bullets). The pair of black tracks are the calorimeter seed tracks projected into the silicon. The silicon pattern recognition algorithm is able to clearly distinguish between the two charge hypotheses by attaching silicon hits to the seed track on the right. The green track is the resulting silicon track.

Electron candidates are first identified based on calorimeter measurements. The energy deposited in the hadron section of the calorimeter is required to be less than $5 \%$ of the energy in the electromagnetic section, and the extra energy in a cone of radius 0.4 around the primary deposition point is required to be less than $10 \%$ of the total. The centroid of the energy clustered in the PES is used to determine the position of the electron candidate in the calorimeter. The position of the primary collision vertex is measured with the other tracks in the event and provides a second point for the electron trajectory. The curvature of the electron trajectory is determined from the transverse energy, $E_{t}=E \sin \theta$, measured by the calorimeter. These two points and the curvature, with appropriate covariance, are used to generate a seed helix for each charge hypothesis.

These seeds are then projected into the silicon where hits are attached using the same pattern recognition algorithm which is used for COT seeded tracks, see Fig. 3. Either of the seed tracks can result in a silicon track if sufficient silicon hits are attached; a minimum of four hits are required for this analysis. If both seed tracks give rise to a silicon track, the one with minimum $\chi^{2} /$ dof is chosen. The efficiency of this calorimeterseeded algorithm is comparable to the COT seeded algorithm.

Correct determination of the charge is important for the asymmetry measurement. 


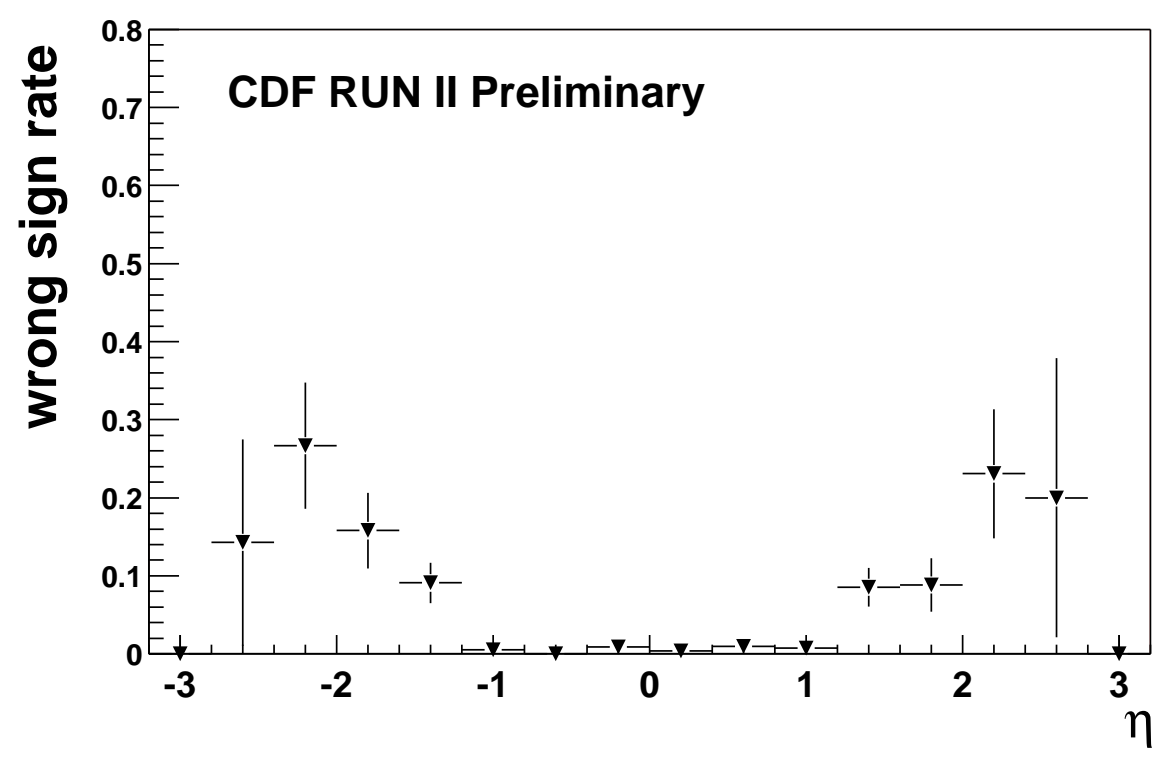

FIGURE 4. Charge mis-identification rate measured with $Z \rightarrow e^{+} e^{-}$data is plotted as a function of pseudo-rapidity.

The charge mis-identification rate of the algorithm is determined from the data using a sample of $Z \rightarrow e^{+} e^{-}$candidates. One of the two leptons is required to be in the central region where its charge is well identified by a matched COT track. That determines the charge of the other lepton which is used to probe the charge mis-identification rate as a function of $\eta$.

Fig. 4 shows the measured charge mis-identification rate as a function of the lepton pseudo-rapidity. In the central region the charge mis-identification is well below $2 \%$. For $1.0 \leq|\eta| \leq 2.0$ it is between $10 \%$ and $15 \%$ rising up to $27 \%$ for $2.0<|\eta| \leq 2.6$. Improvements in the algorithm and the detector alignment will reduce this rate to less than $10 \%$ over the full $\eta$ range, but the performance is already sufficient for use in the asymmetry measurement.

\section{ASYMMETRY MEASUREMENT}

$W \rightarrow e v$ candidate events are selected from two online trigger paths, one for the central region of the detector, $|\eta|<1$, and another for the forward region. The data sample was collected between February and September 2002 and corresponds to an integrated luminosity of $32 \mathrm{pb}^{-1}$. Selected events are required to satisfy the following criteria:

- exactly one electron candidate, passing the criteria described above, with transverse energy $E_{t}>15 \mathrm{GeV}$

- missing transverse energy $E_{t}>30 \mathrm{GeV}$ 


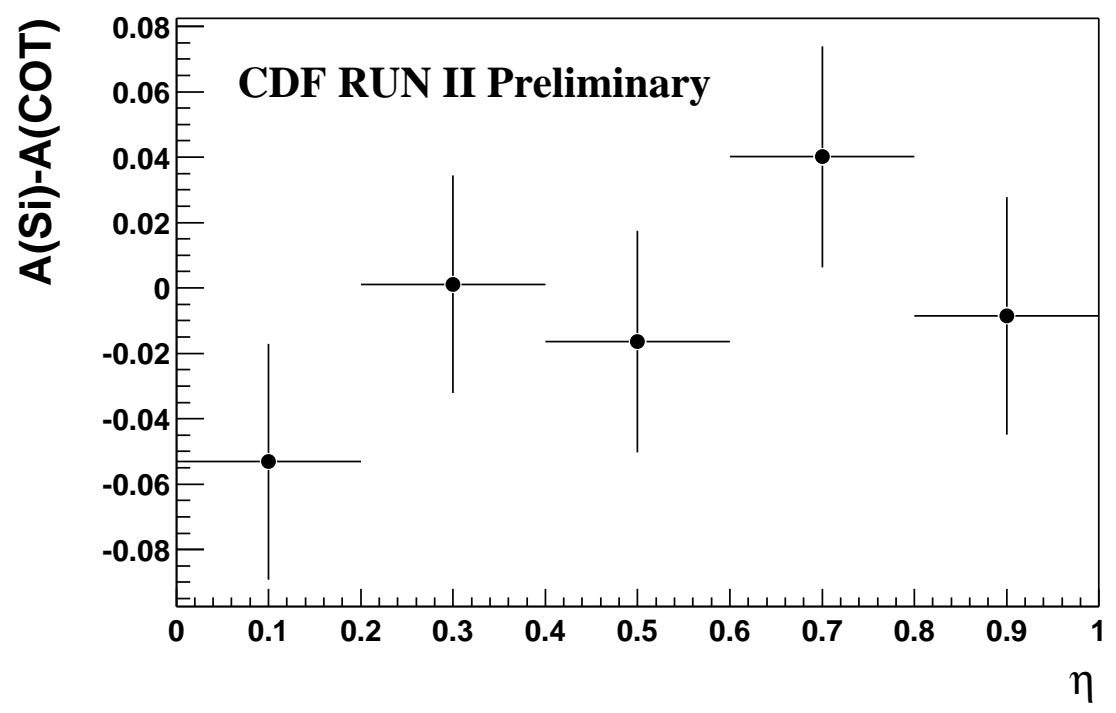

FIGURE 5. Difference between the $W$ charge asymmetry determined with calorimeter seeded silicon tracks and with COT tracks versus pseudo-rapidity.

- transverse mass, $50 \leq M_{t}<100 \mathrm{GeV}^{3}$.

The calorimeter-seeded silicon tracking is used for the charge determination in all $\eta$ regions. In the central region, $|\eta|<1$, the COT tracking information is also available and is used as a cross-check. Fig. 5 shows the difference in that region between the $W$ charge asymmetry measured with the silicon only tracking and with the COT tracking. The two approaches yield consistent results.

Since the data sample collected so far is small, we do not yet consider the actual measured asymmetry values. Rather, they are set to zero to avoid potential bias and will not be unblinded until a larger data sample is collected and systematic studies are completed. Nonetheless, the sensitivity of the measurement can be determined. This is shown in Fig. 6 where the charge asymmetry is plotted as a function of $|\eta|$ but with the central values set to zero. The uncertainties shown include the statistical errors and the systematic uncertainty from the charge mis-identification rate. For $|\eta|>1.6$ the charge mis-identification uncertainty contributes $50 \%$ of the total error. The figure compares this preliminary Run 2 sensitivity to the Run 1 measurement [1] and to three parton density functions, to indicate the range of variation in the parton structure functions at high $\eta$.

With only $32 \mathrm{pb}^{-1}$ of Run 2 data in the electron mode, the sensitivity is not yet competitive with the existing Run 1 measurement obtained with both electrons and muons. However, it can be used to provide a measure of the improvement in sensitivity

\footnotetext{
3 The transverse mass is given by $M_{t}=\sqrt{\left[E_{t}(e)+E_{t}(v)\right]^{2}-\left[\vec{p}_{t}(e)+\vec{p}_{t}(v)\right]^{2}}$, where $\vec{p}_{t}(e)$ and $\vec{p}_{t}(v)$ are the transverse momentum of the electron and the neutrino.
} 


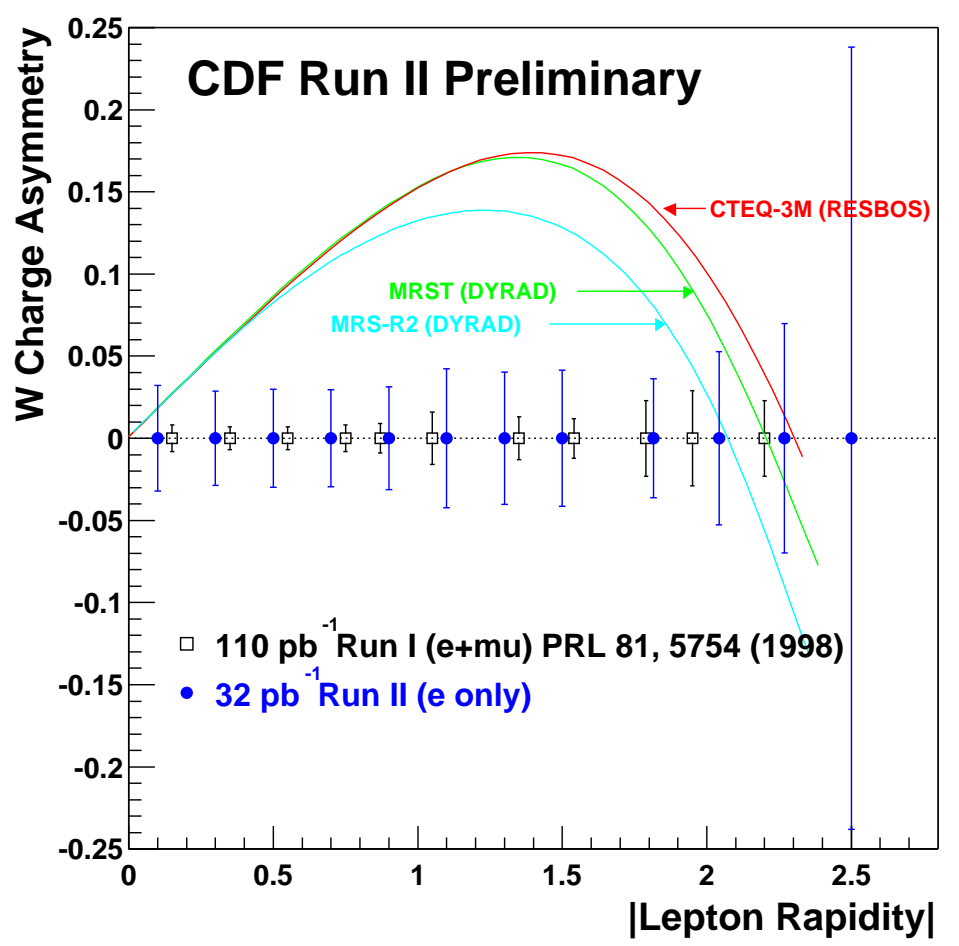

FIGURE 6. $W$ charge asymmetry sensitivity with calorimeter-seeded silicon tracks is compared between Run 2 electrons (bullets) and the Run 1 measurement using both electrons and muons (open squares). The central values of the data points are set to zero because the result will not be unblinded until a larger data sample is collected and systematic studies are completed. The uncertainties shown include the statistical errors and the systematic uncertainty from the charge mis-identification rate.

which will be obtained beyond just the ultimately higher integrated luminosity. This is illustrated in Fig. 77 where the current uncertainties are scaled to a $120 \mathrm{pb}^{-1}$ sample which should be collected in the near future. This projection is a conservative comparison in that it does not include the effect of the muon mode from Run 1 or the ongoing improvements in Run 2 detector performance. Nonetheless, it illustrates the gain in precision which will soon be obtained in the $|\eta|>1$ region to further constrain the $u / d$ ratio in the proton structure function. Furthermore the Run 2 measurement can extend the sensitivity up to up to $|\eta|=2.5$.

\section{ACKNOWLEDGMENTS}

I would like to thank the Division of Particles and Fields of the Mexican Physical Society for the invitation to and the organization of the X Mexican School of Particles and Fields. I thank Joel Goldstein, Joe Incandela, Tim Nelson, Rick Snider and David Stuart for close collaboration and discussions. I thank the Fermilab staff and the technical staff of the participating institutions for their vital contributions. This work was supported 


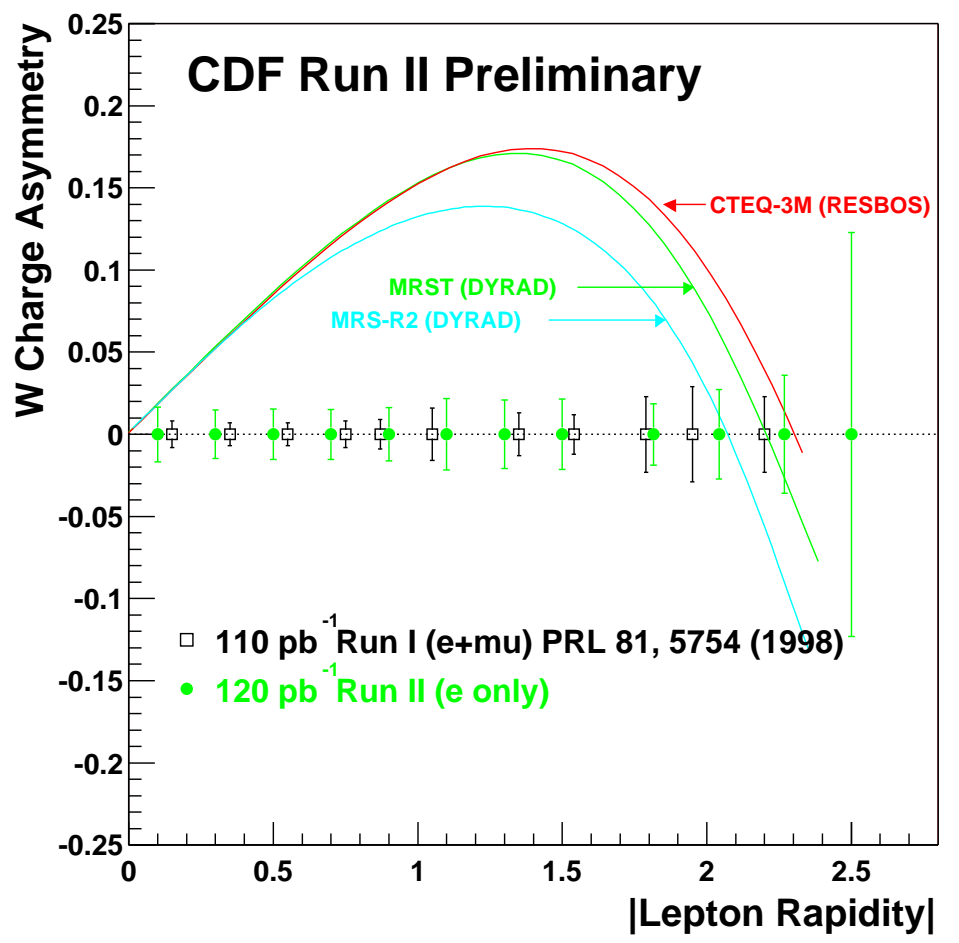

FIGURE 7. A $120 \mathrm{pb}^{-1}$ projection of the Run $2 \mathrm{~W}$ charge asymmetry sensitivity (bullets) is compared to the Run 1 measurement (open squares) as a function of pseudo-rapidity.

by the U.S. Department of Energy and National Science Foundation; the Italian Istituto Nazionale di Fisica Nucleare; the Ministry of Education, Science and Culture of Japan; the Natural Sciences and Engineering Research Council of Canada; the National Science Council of the Republic of China; and the A. P. Sloan Foundation.

\section{REFERENCES}

1. CDF-Collaboration, Phys. Rev. Lett., 81, 5744 (1998).

2. CDF-Collaboration, The CDF II Detector Technical Design Report, Tech. rep., FERMILAB-Pub96/390-E (1996). 